\title{
Autonomous Synchronization of a DMT-VDSL System in Unbundled Networks
}

\author{
Rickard Nilsson, Student Member, IEEE, Frank Sjöberg, Member, IEEE, Mikael Isaksson, \\ John M. Cioffi, Fellow, IEEE, and Sarah Kate Wilson, Senior Member, IEEE
}

\begin{abstract}
We present an algorithm that autonomously synchronizes all DMT-Zipper based VDSL modems in an unbundled access network, solving the problem with nonorthogonal NEXT that appears in systems with unsynchronized modems. The algorithm we present runs autonomously in each VDSL-modem in the central office or in the street cabinet. We determine the other modems' relative frame offsets by exploiting their NEXT signal using the inherent cyclic redundancy found in DMT signals. By estimating the relative frame offsets of the other users, we can adjust a given user's own frame-timing relative to the mean of the others. With our method all modems in the network will be synchronized to within a small fraction of the total DMT frame-length, suppressing the nonorthogonal NEXT to a level far below the background noise-floor. This means that we can achieve the same performance in an unbundled access network without any master clock reference as in a system where all modems are perfectly synchronized using a master clock.
\end{abstract}

Index Terms-Discrete multitone, frame synchronization, subscriber loops.

\section{INTRODUCTION}

$\mathbf{I}^{\mathrm{N}}$ $\mathrm{N}$ THIS PAPER, we describe an algorithm that autonomously frame-synchronizes all modems in a discrete multitone (DMT)-based [1] very high-speed digital subscriber line (VDSL) system [2] using the Zipper duplex method [3]. This algorithm is useful for unbundled access networks where it is difficult to provide a common master clock to all VDSL modems.

The DMT-based Zipper duplex method [3], assigns different subcarriers between the up- and down-stream, splitting the capacity in a flexible and bandwidth efficient manner. To achieve the best possible performance, frame synchronization between all VDSL-modems is required to completely eliminate the near-end crosstalk (NEXT). Without synchronization nonorthogonal NEXT occurs, reducing the bit rate capacity. Most of the nonorthogonal NEXT can be suppressed by

Manuscript received April 2, 2001; revised December 7, 2001. This paper was presented in part at the International Conference on Communications 2001, Helsinki, Finland.

R. Nilsson is with Telecommunications Research Center Vienna (FTW), Donau-City-Strasse 1/3, A-1220 Vienna, Austria.

F. Sjöberg is with the Division of Signal Processing, Luleå University of Technology, SE - 97187 Luleå, Sweden and also with Telia Research AB, Aurorum 6, SE-977 75 Luleå, Sweden.

M. Isaksson is with UpZide Labs AB, Aurorum 7, SE-977 75 Luleå, Sweden.

J. M. Cioffi is with the Electrical Engineering Department, Stanford University, Stanford, CA 94305-9515 USA.

S. K. Wilson is with ArrayComm, San Jose, CA 95131-1014 USA.

Publisher Item Identifier S 0733-8716(02)05383-0. grouping the subcarriers used in each transmission direction into larger frequency bands and using pulse-shaping and windowing on the time-domain DMT-signal [4]. However, some nonorthogonal NEXT will still remain, resulting in signal-to-noise (SNR)-loss at the subcarriers close to the bandedges between the up- and downstream bands.

Synchronization may be a problem in the deregulated telecommunication market due to unbundling of the twisted pairs in the access network. In the unbundled environment, wires in the same cable-bundle can be connected to modems located in physically different central offices (COs), or street cabinets that belong to different operators [5]. Unbundled wires make it difficult for the modems in the network to share a common master clock for synchronization. The decentralized algorithm we present runs autonomously in each VDSL-modem at the $\mathrm{CO}$ side, requiring no extra wiring for synchronization.

To solve the synchronization problem we have developed a simple frame synchronization algorithm using the cyclic extensions of the DMT signals. Using a cyclic prefix for time offset estimation has been proposed by many others before [6]-[8]. The difference here is that we consider the system-wide synchronization of all modems at the CO-side, not the line synchronization between two modems on the same line as in [6]-[8]. Rather than estimating the start-time of a user's own DMT frame, we use the redundancy of the other modems' to estimate their frame offsets relative to that of the user-of-interest. The user-of-interest's frame timing is then adjusted toward the mean of the others, which results in a global frame synchronization of the VDSL-system.

The modems do not have to be perfectly synchronized to achieve the same bit rate performance as with perfect synchronization. If the NEXT is suppressed far below the background noise floor, the loss of SNR is negligible and consequently there is essentially no performance loss. Our simulations show that the algorithm we present gives sufficient synchronization to achieve the same performance as a system using a master clock.

\section{SYSTEM MODEL}

The Zipper VDSL duplex method is based on DMT modulation. The duplexing is performed digitally ${ }^{1}$ by assigning each subcarrier to either the up- or downstream direction to avoid NEXT and near echoes. Since VDSL is baseband communication the DMT modulator shall generate a real-valued signal. One

${ }^{1}$ Zipper is also known as digital duplexing. 


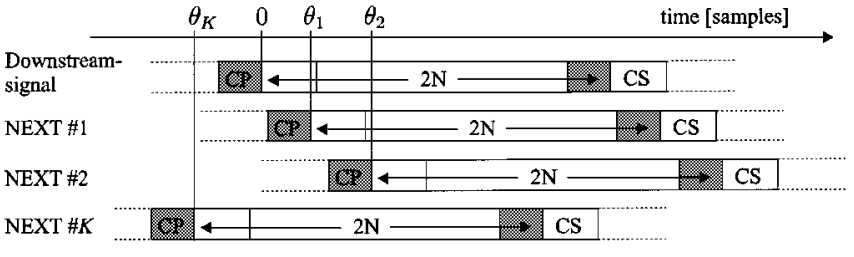

Fig. 1. The time-domain downstream DMT-signal and the relative offsets, $\theta_{k}$, to the NEXT signals.

DMT symbol transmitted in the upstream direction can be modeled as

$$
x_{\uparrow}(t)=\left\{\begin{array}{c}
\operatorname{Re}\left\{\sum_{k \in \mathcal{I}_{\uparrow}} X_{k} e^{\left(j 2 \pi k f_{s} / 2 N\right) t}\right\} \\
t \in\left[-\frac{C P}{f_{s}}, \frac{2 N+C S}{f_{s}}\right] \\
0, \quad \text { otherwise }
\end{array}\right.
$$

where $\mathcal{I}_{\uparrow}$ is the index set for the upstream carriers, $N$ is the total number of subcarriers, $C P$ is the length (in samples) of the cyclic prefix, $C S$ is the length of the cyclic suffix, $X_{k}$ is the QAM-data, and $f_{s}$ is the sampling frequency. The cyclic suffix is, similar to the cyclic prefix [9], a cyclic extension of the DMT-symbol at the end of the symbol. The cyclic suffix is required to make the near echo orthogonal to the received signal. Its length has to be at least as long as the propagation delay of the longest wire in the system. If all modems in the network are synchronized the NEXT will also be orthogonal [3].

The received signal on one line in the central office is a sum of four parts; the desired signal, the near echo, the NEXT from other modems and additive white Gaussian noise (AWGN) and far-end crosstalk (FEXT). This is represented for modem $k$ by

$$
\begin{aligned}
r_{k}(t)=x_{\uparrow, k}(t) * h_{\uparrow, k}(t)+x_{\downarrow, k}(t) & * h_{\mathrm{NE}, k}(t) \\
& +v_{\mathrm{NEXT}, k}(t)+n_{k}(t)
\end{aligned}
$$

where the arrows indicate the transmission direction and $n_{k}(t)$ is AWGN and other crosstalk. Assuming that the modems are not synchronized to a common master clock the NEXT for modem $k, v_{\mathrm{NEXT}, k}(t)$, can be modeled by

$$
v_{\mathrm{NEXT}, k}(t)=\sum_{l=1, n \neq k}^{K} x_{\downarrow, l}\left(t-\left(\theta_{l}-\theta_{k}\right)\right) * h_{\mathrm{NEXT}}^{l \rightarrow k}(t)
$$

where $K$ is the number of active VDSL modems, $\theta_{k}$ denotes the frame offset in time of modem $k$ (see Fig. 1), and $h_{\mathrm{NEXT}}^{l \rightarrow k}(t)$ is the NEXT transfer function from line $l$ to line $k$. Unsynchronized modems result in nonorthogonal NEXT that will appear as sidelobe leakage, which is strongest at the band edges between the up- and downstream. The problem with residual NEXT becomes more pronounced for longer loops where the received signal suffers from attenuation.

A large amount of the nonorthogonal NEXT can be suppressed efficiently by using windowing and pulse-shaping on the time domain DMT-signals and by grouping subcarriers used in the same transmission direction into larger frequency-bands [4]. Using that, almost the same capacity can be achieved without any synchronization of modems on different lines. However, the remaining NEXT close to the bandedges results in SNR-loss, which necessitates the grouping of subcarriers into larger bands. Accordingly, it is not possible to use every other subcarrier for the up- and downstream direction (full zippering) without any network synchronization.

We have developed a simple method, that can synchronize all line-pairs in the network without a master clock. Assuming that each modem at the CO-side can estimate the other modems' frame offsets $\theta_{k}$, synchronization is performed by adjusting the user-of-interest's frame timing toward the mean of the other modems' offsets. In Section IV we show how the cyclic extensions can be used to derive an estimate of the other modems' frame offsets $\theta_{k}$, and Section III below describes how the timing adjustment can be implemented. In this paper, we do not address the line synchronization that needs to be established whether our method or a master clock is used.

\section{Frame Timing AdJustment}

In this section, we describe a simple control method for synchronizing the frame timing. The control algorithm is robust and suitable for VDSL low-cost implementation, though not optimal (in the sense of minimizing the frame offset's variance). The goal of the algorithm is to adjust the frame time offset of an individual modem to the mean value of the other modems' frame offsets relative to the user-of-interest's, denoted $\theta_{\mathrm{av}, k}$ for modem $k$. If all modems follow this procedure their frame timing will converge to their common mean. This is shown in the Appendix.

For simplicity and stability we use a zeroth order controller to adjust the frame-timing. Consider one modem. If the estimated average frame offset of the other modems is $\hat{\theta}_{\mathrm{av}, k}$, we simply adjust our user-of-interest's frame clock by $c \hat{\theta}_{\mathrm{av}, k}$ samples

$$
\theta_{k}[n+1]=\theta_{k}[n]+c \hat{\theta}_{\mathrm{av}, k}[n], \quad 1 \leq k \leq K
$$

where $\theta_{k}[n]$ is the absolute offset for modem $k$ at time $n$, and $c$ is the gain constant. To ensure stability and convergence the gain has to be in the range $0<c<2(K-1) / K$, as shown in the Appendix. Furthermore, the drift of the common offset after convergence, relative to a perfect reference clock, will depend on the average sampling clock offsets of all modems, also shown in the Appendix. Because averaging improves the frame-offset estimate, the observation interval over which $\hat{\theta}_{\mathrm{av}, k}$ is determined is usually many DMT-frames long. The updating according to (4) is performed once per observation interval. We choose not to control the frequency of the sampling clock since, even if there is no frequency offset, a small additive bias in the estimates would eventually saturate the voltage controlled oscillator and break down the control scheme.

The gain, $c$, affects the convergence speed of the system as well as the noise bandwidth. A smaller value of $c$ provides slower convergence but makes the system more robust to estimation errors. Another way to make the controller more robust to noisy estimates is to limit the number of samples that the clock is allowed to change, i.e., $\left|c \hat{\theta}_{\mathrm{av}, k}[n]\right| \leq \Delta_{\max }$. In general, we do not believe the convergence speed is of great importance, since a case where all or several modems 
are unsynchronized would be a rare event, e.g., after a power failure. In a normal start-up procedure a modem should be silent until it has determined the other modems frame timing to avoid creating nonorthogonal NEXT in the binder. Only once the proper frame timing is determined, by calculating $\hat{\theta}_{\mathrm{av}, k}$, the modem can start to transmit synchronously with the others.

By using a cyclic extension that is $B$ samples longer than that required for a perfectly synchronized system, we get a synchronization margin of $\pm B / 2$ samples. This means that if $\left|\theta_{l}-\theta_{k}\right|<$ $B$ for all $l$ and $k$ the NEXT will be orthogonal and the same performance is achieved as with perfect synchronization. The draw back is that a longer cyclic extension reduces the bandwidth efficiency. However, in our simulations, shown in Section V, we have used the cyclic extension length defined in the ETSI VDSL specifications [10].

The frame timing adjustment is implemented as a skip/stuff scheme [11]. This is performed simultaneously in both modems on the same line in order to prevent changes (rotations) in the channel estimates that otherwise can cause symbol errors. The number of samples to skip/stuff are sent to the modem at the customer side through the control channel. Stuffing samples, (which makes the DMT-frame longer) never affects the orthogonality, but skipping too many samples at one instant can affect the orthogonality of the received signal. However, the scheme can be implemented to skip one or a few samples several consecutive frames instead of skipping many samples once.

\section{OFFSET ESTIMATION}

The control algorithm described above is based on each modem at the central office having an estimate of the other modems' average frame-offset relative to the user-of-interest's

$$
\theta_{\mathrm{av}, k}=\frac{1}{K-1} \sum_{l=1, l \neq k}^{K}\left(\theta_{l}-\theta_{k}\right) \text {. }
$$

The NEXT component $v_{\text {NEXT, } k}(t)$ in the received signal $r_{k}(t)$ in (2) contains information about the other modems offsets $\theta_{l}, l \neq k$, due to the redundancy in the cyclic extensions. The problem of calculating $\theta_{\mathrm{av}, k}$ lies in extracting all $\theta_{l}$, $\{l \mid 1 \leq l \leq K, l \neq k\}$ from $r_{k}(t)$.

Using the redundancy in the cyclic extension of DMT signals for synchronization has been proposed in many papers [6], [7], [12]. For one received real-valued DMT signal the maximum-likelihood (ML) estimate of the time offset $\theta$ of the desired upstream signal, assuming an ideal AWGN channel, is given by [7]

$$
\hat{\theta}_{M L}=\arg \max _{\theta}\{\Lambda(\theta)\}
$$

where

$$
\begin{aligned}
\Lambda(\theta)=\sum_{l=0}^{C E-1} r(\theta+l) & r(\theta+l+2 N)-\frac{1}{2} \frac{\mathrm{SNR}}{\mathrm{SNR}+1} \\
& \cdot \sum_{l=0}^{C E-1} r^{2}(\theta+l)+r^{2}(\theta+l+2 N)
\end{aligned}
$$

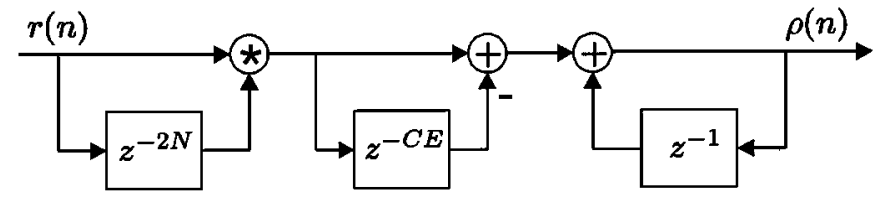

Fig. 2. Correlator block diagram.

where $C E$ is the total length of the cyclic extensions. If the observation interval of the signal spans several DMT-frames the ML-estimate is derived by averaging the log-likelihood function prior to searching for the maxima

$$
\hat{\theta}_{\mathrm{ML}}=\arg \max _{\theta}\left\{\sum_{m=0}^{M-1} \Lambda_{m}(\theta)\right\}
$$

where $M$ is the number of complete DMT-frames in the observation interval. Larger $M$ improves the estimate, but the choice of $\Delta_{\max }$ puts an upper limit on $M$ due to the averaging delay and the clock drift. If the frame timing drifts more than $\Delta_{\max }$ during $M$ frames our synchronization scheme will not work.

For an increasing number of observed DMT-frames, the second sum in (7), the energy terms, converges to a constant value that is independent of $\theta$. Therefore, we do not loose any substantial information by using only the correlation part of the signal,

$$
\rho(\theta)=\sum_{l=0}^{C E-1} r(\theta+l) r(\theta+l+2 N) .
$$

By using only the correlation part we reduce the complexity and making it more practical when we have a sum of several DMT-signals. Fig. 2 shows a block diagram of how to calculate the correlation signal $\rho(\theta)$ efficiently.

Deriving the ML-estimate for several offsets $\theta_{k}$ given a sum of several different DMT-signals, as in the VDSL-case (2), is straight-forward and analogous to the derivation in [7]. However, the solution is impractical, e.g., we need to know the signal energies of all signals, and it is also computationally very complex. Looking at the auto-correlation signal (9) for modem $k$ when the received signal is a sum of signals as in (2) we find that the expected value of $\rho_{k}(\theta)$ is

$$
E\left\{\rho_{k}(\theta)\right\}=\sum_{l=1}^{K} E\left\{\rho_{k, l}(\theta)\right\}
$$

where each $\rho_{k, l}(\theta)$ corresponds to the correlation caused by modem $l$ s downstream signal on modem $k$ s received signal (at the same end of the line). This means that each modems' correlation signal contains information about all modem's signal offsets, $\theta_{k}$.

Fig. 3 shows two examples of the correlation signal $\rho_{k}(\theta)$ averaged over $M=50$ DMT-frames, for a sum of three DMT-signals. It consists of superimposed correlation peaks corresponding to two NEXT-signals and the user-of-interest's near echo. The strong near echo from the downstream signal will result in a peak centered closely to zero $\theta_{N E} \approx 0$. When the individual correlation peaks are separated we can use the correlation peaks from the NEXT signals to determine the $\theta_{k} \mathrm{~s}$, 


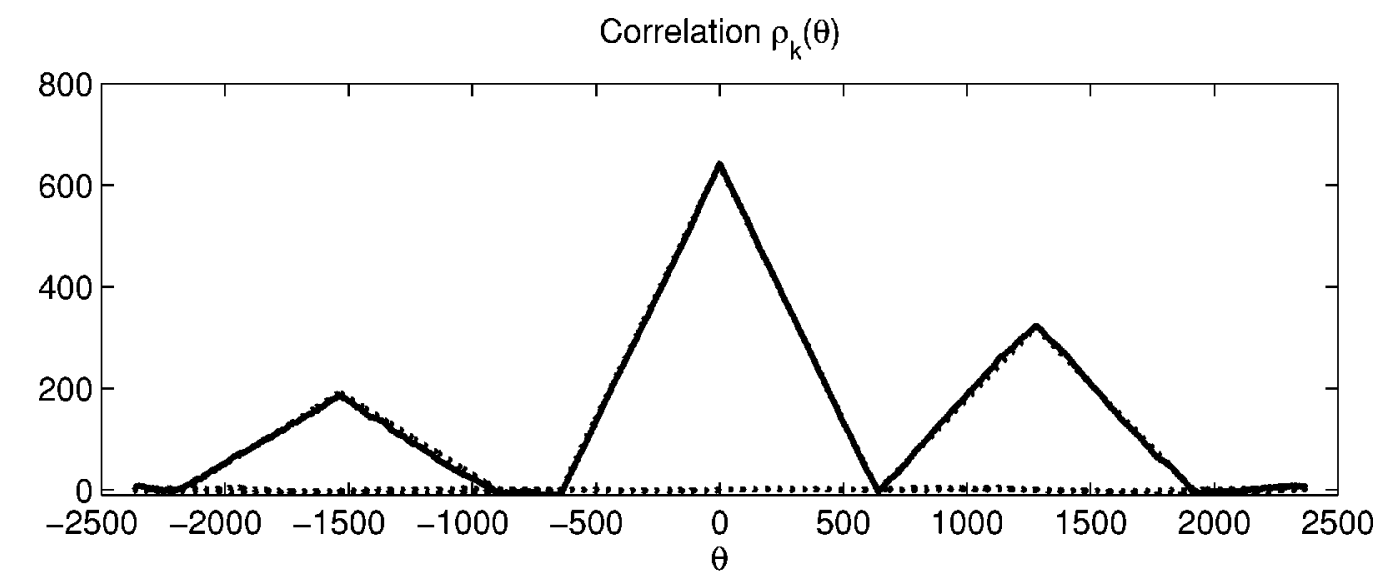

(a)

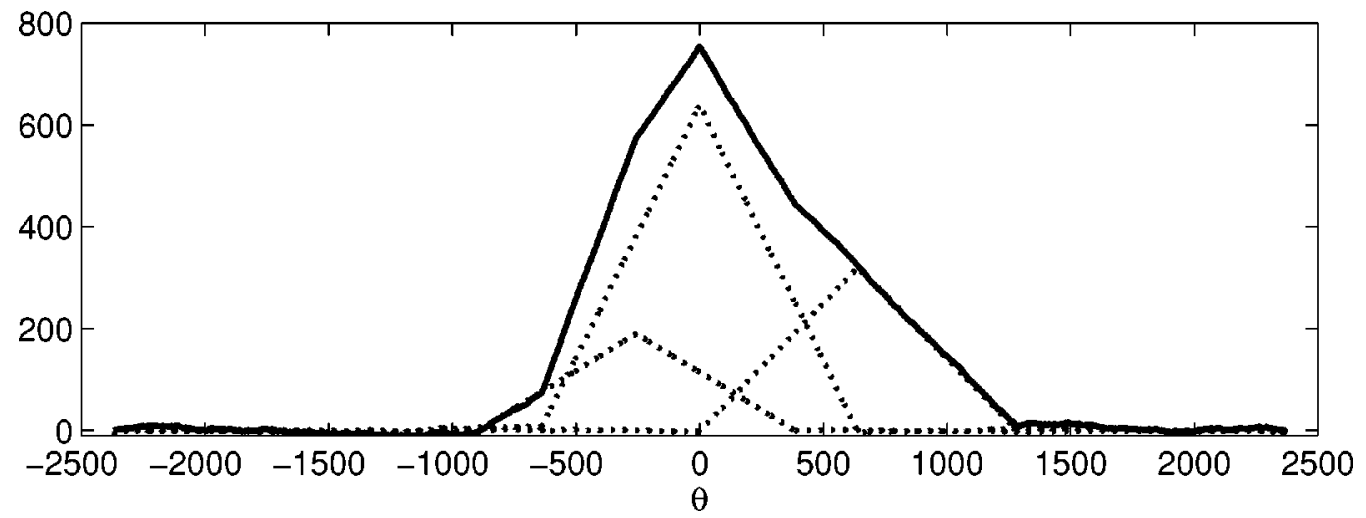

(b)

Fig. 3. Correlation signal, $\rho_{k}(\theta)$, for a sum of three DMT-signals. (a) Case where the correlation peaks are separated. (b) Case where the correlation peaks are superimposed.

as shown in the top figure in Fig. 3. The NEXT correlation peaks will vary in height and position depending on their relative interconnections through the NEXT coupling between the lines and frame timing, respectively.

When the modems are roughly synchronized the individual correlation peaks will be superimposed into one large peak, as shown in the bottom figure in Fig. 3. Then, it is difficult to distinguish each individual peak. But, since we are only interested in the average of the other modems offsets, $\theta_{\mathrm{av}, k}$, we avoid that problem by calculating the center of gravity (or mean-value) of the correlation signal as an estimate of $\theta_{\mathrm{av}, k}$

$$
\hat{\theta}_{\mathrm{av}, k}=\frac{\sum_{\theta=-P}^{P} \theta \rho_{k}(\theta)}{\sum_{\theta=-P}^{P}\left|\rho_{k}(\theta)\right|} \approx \theta_{\mathrm{av}, k}
$$

where $P$ and $-P$ represents the upper- and lower limits of the estimated offsets, respectively. For this application, it will produce a robust and sufficiently accurate estimate of $\theta_{\mathrm{av}, k}$ even when all peaks are superimposed, which we verify by simulations in Section V. If the strengths of the NEXT-signals differ, this estimate can be seen as a weighted average of the individual $\theta_{l}$ s. If some modem's offset should be outside the range of the averaging, $\left|\theta_{l}\right|>P$, it can easily be detected by its off-centered correlation peak.
TABLE I

SYSTEM PARAMETERS

\begin{tabular}{l|l}
\hline Number of subcarriers, $N$ & 4096 \\
\hline Total length of cyclic extensions, $C E$ & 640 samples \\
\hline Sampling frequency, $f_{s}$ & $35.2 \mathrm{MHz}$ \\
\hline AWGN-level & $-140 \mathrm{dBm} / \mathrm{Hz}$ \\
\hline Cable type & $1000 \mathrm{~m} \mathrm{TP2}$ \\
\hline
\end{tabular}

It should be noted that contributions from the near echo and upstream signals can be assumed to be known at the receiver in advance and therefore they can be removed by subtraction to unveil the desired NEXT correlation peaks. The contribution from the received upstream signal can also be attenuated sufficiently by using a low order high-pass filter since the upstream signal has lowpass characteristics compared to the desired NEXT signals. This filter also reduces the NEXT contributions from other services such as ADSL, HDSL etc. since they operate mainly below $1 \mathrm{MHz}$.

Another possible source of disturbance is upstream FEXT from other users, particularly from modems on short wires. But, since power backoff will be used to equalize the capacity distribution for all wire pairs in a binder [13], [14] neither long nor short wires will create strong FEXT. For example, if the reference noise power backoff method [13], [15], [16] is used, the received FEXT on each line will only be a few decibels higher 


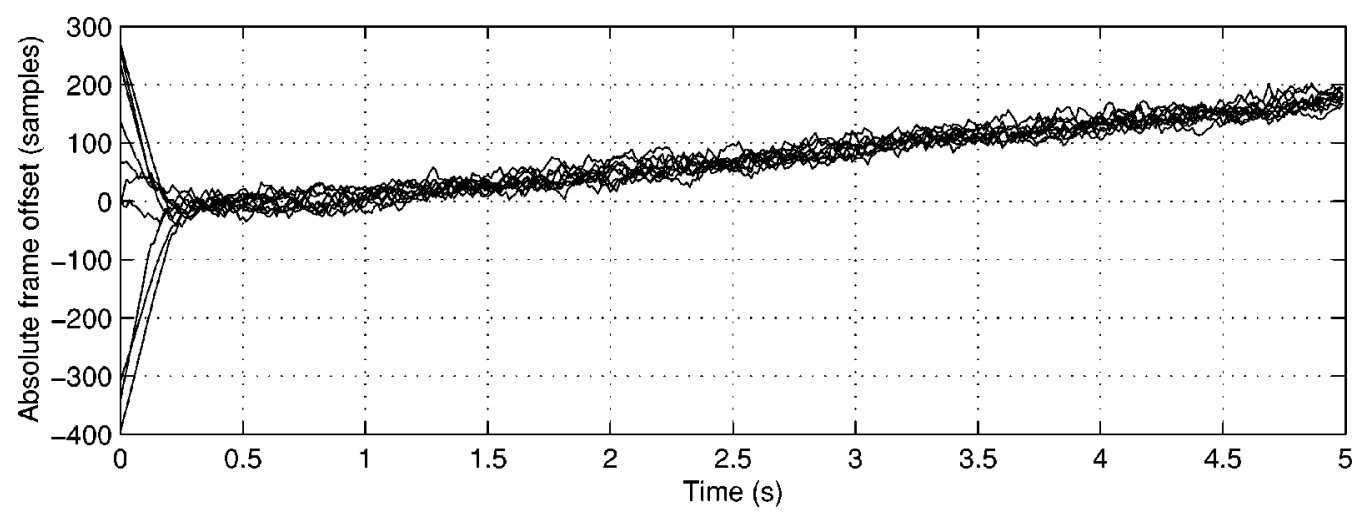

(a)

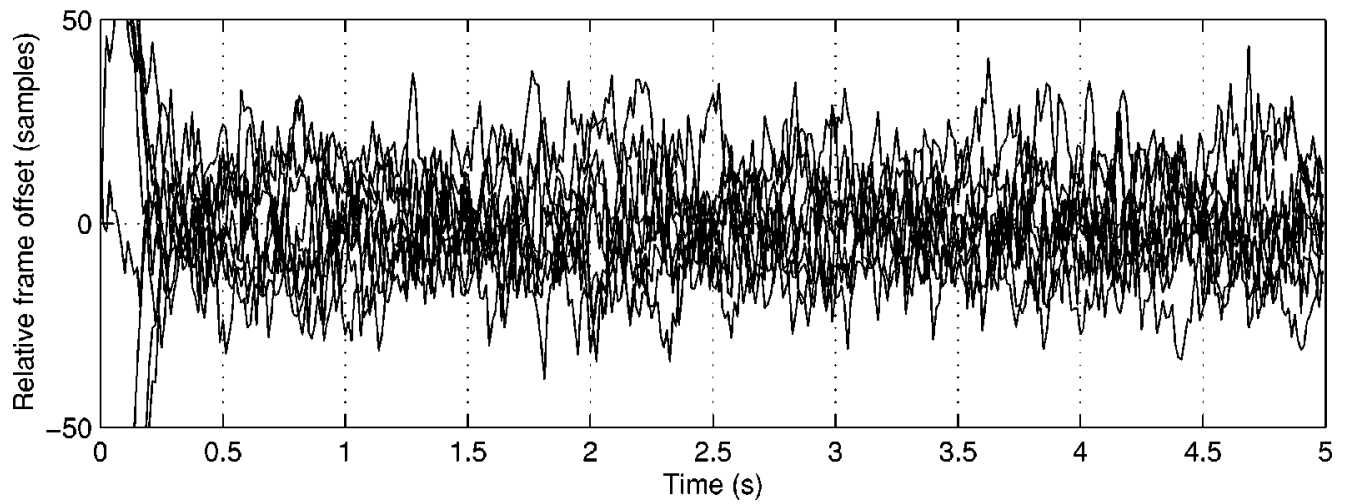

(b)

Fig. 4. Simulated frame offset for ten modems with free running oscillators using the frame synchronization method. (a) Absolute offset. (b) Offset relative to the mean of the offsets.

than the background noise-floor. Thus, this will not be a problem in practice.

\section{SYSTEM SimULATIONS}

We have simulated the synchronization method in a Zipper-VDSL system with 10 users. Each users' modem has a free running sampling clock that can differ up to $\pm 10 \mathrm{ppm}$. This frequency offset will not introduce any significant nonorthogonality itself [11] if the modems are time synchronized, but without system-wide synchronization it makes the DMT-framing drift, which results in nonorthogonal NEXT unless our method is used.

Our channel model is based on a 0.5 -mm-diameter wire called the TP2-wire [17], and we have used the crosstalk models defined by FSAN [2]. ${ }^{2}$ The near-echo channel is modeled as a flat attenuation of $20 \mathrm{~dB}$. We have used the same impulse response for all NEXT-couplings but with different attenuation (up to $6 \mathrm{~dB}$ ) between different modems. This reflects the variations in the NEXT-coupling that exist between different wire pairs. For example, adjacent wires in the cable will experience more cross-talk than wires that are further apart. Table I lists some of the system specific parameters that are selected from the ETSI VDSL standard [10].

We used $M=50$ frames for averaging when estimating the mean-offsets, which corresponds to 80 updates per second with the selected system parameters. For the estimation we selected

${ }^{2}$ Full service access networks (FSAN) is a group of telephone operators. the averaging range to $P=640$ samples in (11). The adjustment was limited to $\Delta_{\max }=20$ samples and the gain constant was chosen to $c=0.3$. The $\pm 10 \mathrm{ppm}$ offset of the oscillators corresponds to a drift of \pm 5 samples per iteration ( 50 frames), or 400 samples/s. The cut-off frequency of the high-pass filter was $4 \mathrm{MHz}$. In the simulation we let the modems start with random starting offsets between \pm 400 samples relative to a fixed reference. Note, this does not represent a normal start-up, as described in Section III, but rather a reacquisition after, for example, a power failure.

Fig. 4 shows the trajectories of all modems' frame offset. The top figure shows absolute offsets and the bottom figure shows relative offsets. The frame offsets converge quite quickly and the maximum difference, in steady state operation, is always less than 100 samples. Fig. 5 shows the estimated offsets, $\hat{\theta}_{\mathrm{av}, k}$, for all 10 modems as a function of time. The common trajectory after convergence is not flat since the frequency offsets of the oscillators are not adjusted in our control algorithm.

We have evaluated the bit rate performance for two different choices of synchronization margin $B$ (cf. Section III), 100 samples and five samples, since the performance depends on this parameter. The margin is achieved by partitioning the total cyclic extension $(C E=640)$ differently between the prefix and the suffix, since the length of the cyclic extension defined in the ETSI standard [10] gives room for a synchronization margin with 1000 meter long wires. However, a margin of 100 samples could also be interpreted as a reduction in efficiency from $93.4 \%$ to $92.2 \%$. 


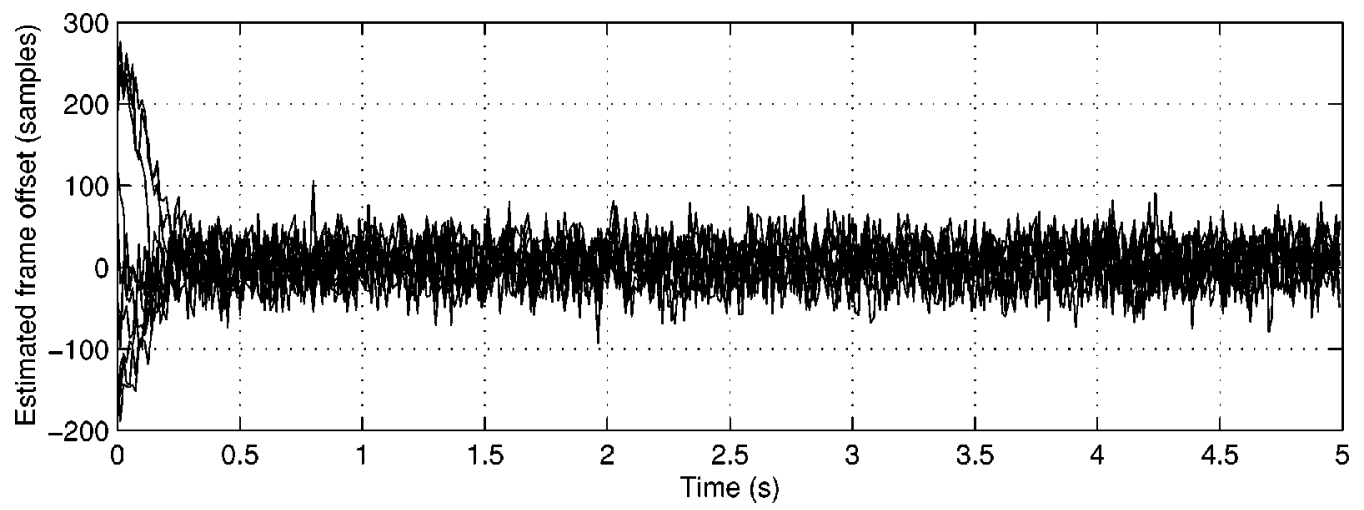

Fig. 5. Estimated mean-offset, $\hat{\theta}_{a v, k}$, for each of the ten modems.

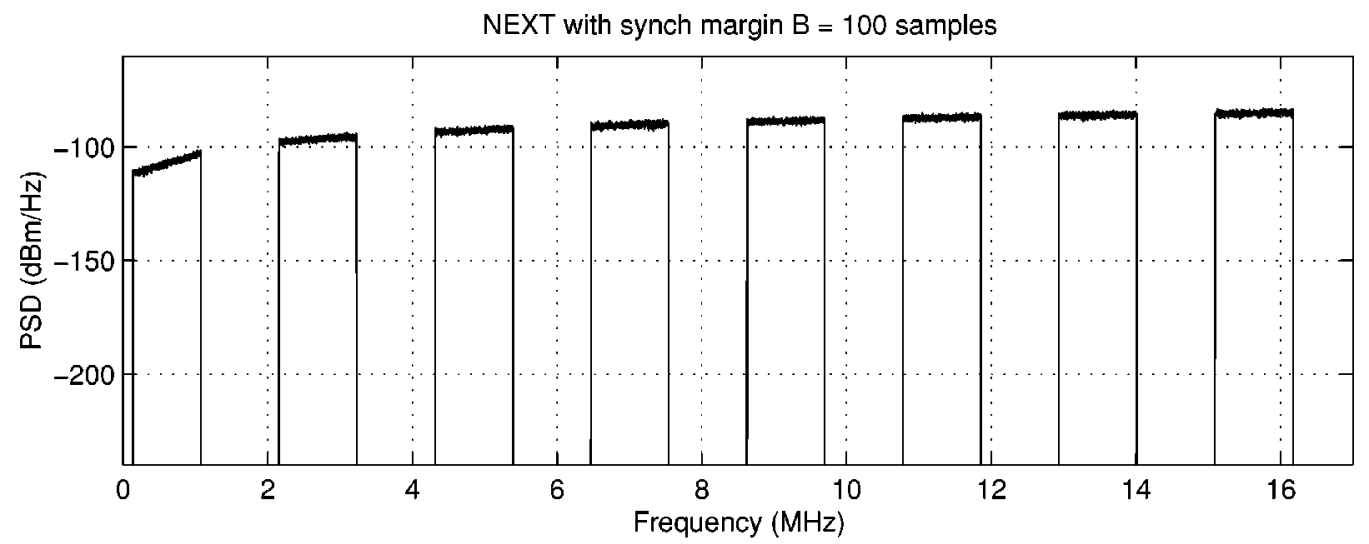

(a)

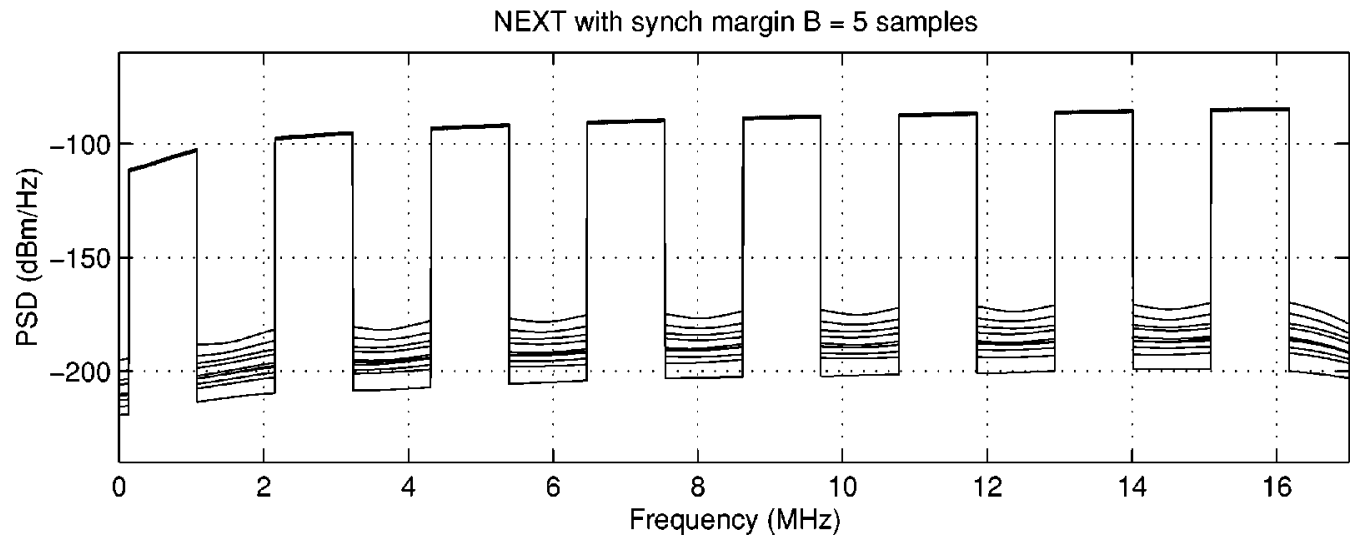

(b)

Fig. 6. NEXT levels for ten modems with a synchronization margin of (a) $B=100$ samples and (b) of $B=5$ samples. The gaps in the curves represent the upstream bands.

Fig. 6 shows the average NEXT, after convergence $(0.5 \mathrm{~s})$, at the central office for the two different cases. In the top figure the synchronization margin is $\pm 50(B=100)$ samples, and in the bottom figure it is $\pm 2.5(B=5)$ samples. For the first case, the NEXT is completely orthogonal in the upstream bands, represented by the gaps in the NEXT curves. This results in zero SNR-loss compared to a perfectly synchronized system, as shown in the top figures in Fig. 7. The left hand figures actually contain 11 SNR curves (10 simulated modems and one perfect synchronization reference) but the curves are indistinguishable. To actually view the performance differences, the SNR-losses are plotted in the right hand figures (note the scale). In the second case, represented by the bottom figure in Fig. 6, some nonorthogonal NEXT appears, but the power spectral density is well below the background noise floor at $-140 \mathrm{dBm} / \mathrm{Hz}$. The corresponding SNR-loss is very small, as shown in the bottom figures in Fig. 7. Consequently, a system with this synchronization method can achieve the same bit rate performance as a system with perfect synchronization. For both cases, the bit rate performance is $15.628 \mathrm{Mbps}$ in the upstream for all modems, using an SNR-gap of $9.8 \mathrm{~dB}$ (for symbol error rate $10^{-7}$ ) [18] and 6-dB system margin. This is the same as with perfect synchronization. 

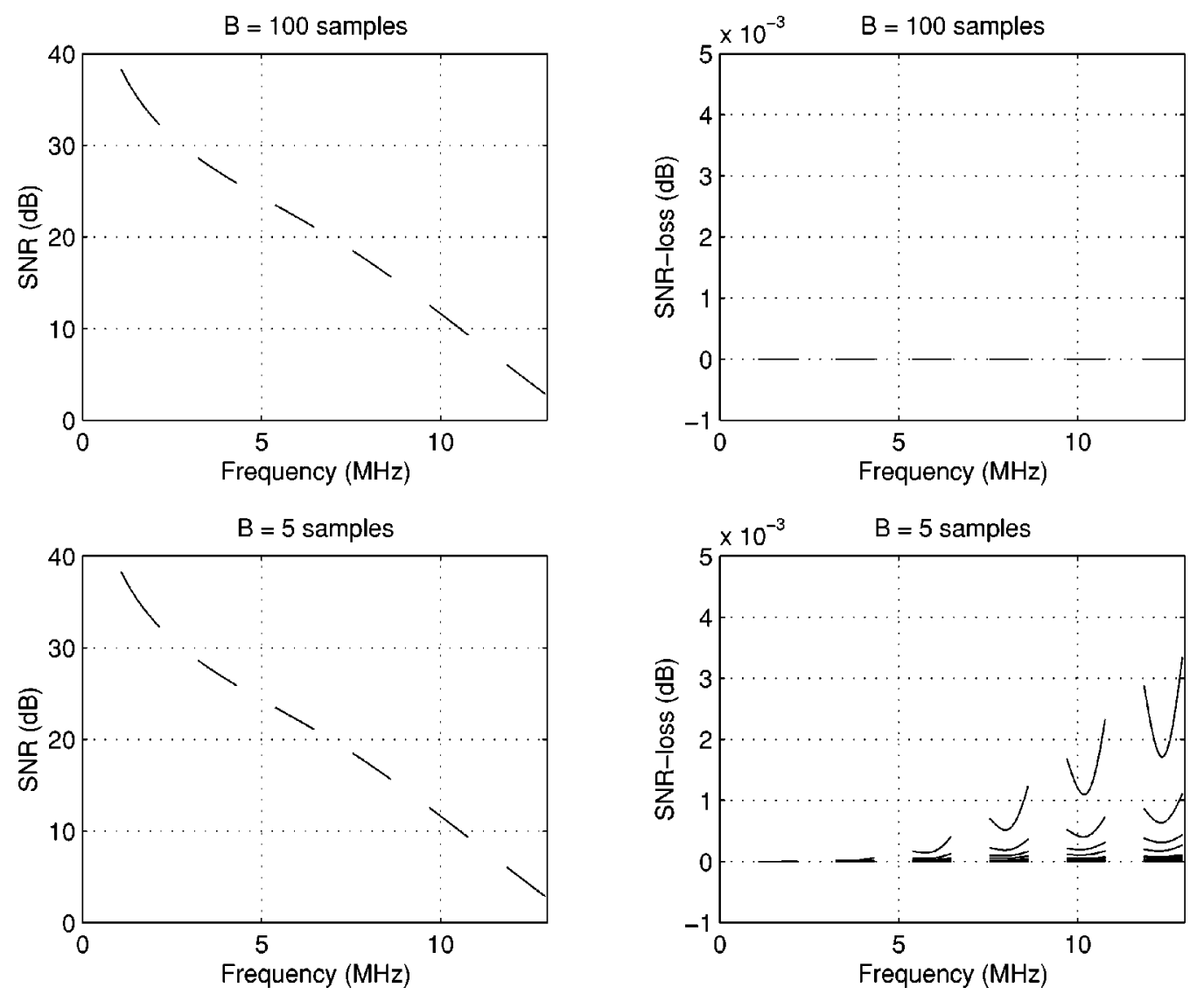

Fig. 7. SNR (left hand) and SNR-loss (right hand) in the upstream for ten modems with a synchronization margin of $B=100$ samples (above) and of $B=5$ samples (below). The gaps in the curves are the downstream bands.

\section{SUMMARY}

We have derived a simple method for achieving system wide synchronization of all modems in a VDSL-system using the Zipper duplex method. This is useful in situations when a master reference clock cannot be used, e.g., in unbundled access networks. The method uses the cyclic extensions inherent in the DMT-signals to estimate the average frame offset of the other NEXT-generating users that share the same cable bundle. The estimated frame offset is used to adjust the user-of-interest's frame timing toward the others. This will synchronize all modems and suppress the NEXT.

Simulations of the method for a Zipper-VDSL system with ten users show that the frame timing can be synchronized to within a small fraction of the total DMT frame length. The residual nonorthogonal NEXT-level is then suppressed far below the background noise-floor. Therefore, we achieve the same performance as with a perfectly synchronized system but without using a common master clock.

\section{APPENDIX}

Here, we calculate the convergence conditions of the algorithm and its steady state characteristics. The update equation for the frame offset $\theta_{k}[n]$, for modem $k$, is

$$
\theta_{k}[n+1]=\theta_{k}[n]+c \hat{\theta}_{\mathrm{av}, k}[n]+d_{k}, \quad 1 \leq k \leq K
$$

where $c$ is a constant feedback gain and $d_{k}$ represents a small clock-drift (which is assumed to be constant in time for each modem). $\hat{\theta}_{\mathrm{av}, k}[n]$ is an estimate of the average offset of the other modems relative to the user-of-interest's

$$
\hat{\theta}_{\mathrm{av}, k}[n]=\frac{1}{K-1} \sum_{l=1, l \neq k}^{K}\left(\theta_{l}[n]-\theta_{k}[n]\right)+v_{k}[n]
$$

where $v_{k}[n]$ represents the estimation error for modem $k$ at time $n$.

By defining the vector $\theta[n] \triangleq\left[\theta_{1}[n] \quad \theta_{2}[n] \cdots \theta_{K}[n]\right]^{T},(12)$ can be expressed in matrix notation as

$$
\begin{aligned}
\boldsymbol{\theta}[n+1]= & \boldsymbol{\theta}[n]+\hat{\boldsymbol{\theta}}_{\mathrm{av}}[n]+\mathbf{d} \\
= & \boldsymbol{\theta}[n]+c\left(\frac{1}{K-1}(\mathbf{1}-\mathbf{I}) \boldsymbol{\theta}[n]-\boldsymbol{\theta}[n]\right) \\
& +c \mathbf{v}[n]+\mathbf{d} \\
= & \left(\frac{c}{K-1}(\mathbf{1}-\mathbf{I})+(1-c) \mathbf{I}\right) \boldsymbol{\theta}[n]+c \mathbf{v}[n]+\mathbf{d} \\
= & \mathbf{A} \boldsymbol{\theta}[n]+c \mathbf{v}[n]+\mathbf{d} \\
= & \mathbf{A}^{n+1} \boldsymbol{\theta}[0]+c \sum_{k=0}^{n} \mathbf{A}^{k} \mathbf{v}[n-k]+\left(\sum_{k=0}^{n} \mathbf{A}^{k}\right) \mathbf{d}
\end{aligned}
$$


where $\mathbf{I}$ represents the identity matrix, $\mathbf{1}$ is a matrix with one in all elements, $\mathbf{v}[n]=\left[v_{1}[n] \cdots v_{K}[n]\right]^{T}$, and $\mathbf{d}=\left[d_{1} \cdots d_{K}\right]^{T}$. The matrix $\mathbf{A}$ is

$$
\mathbf{A}=\left[\begin{array}{cccc}
1-c & \frac{c}{K-1} & \cdots & \frac{c}{K-1} \\
\frac{c}{K-1} & 1-c & \ddots & \vdots \\
\vdots & \ddots & \ddots & \frac{c}{K-1} \\
\frac{c}{K-1} & \cdots & \frac{c}{K-1} & 1-c
\end{array}\right] .
$$

Diagonalizing $\mathbf{A}$ and using that in (18) yields

$$
\begin{array}{r}
\boldsymbol{\theta}[n+1]=\mathbf{Q} \Lambda^{n+1} \mathbf{Q}^{H} \boldsymbol{\theta}[0]+c \sum_{k=0}^{n} \mathbf{Q} \Lambda^{k} \mathbf{Q}^{H} \mathbf{v}[n-k] \\
+\mathbf{Q}\left(\sum_{k=0}^{n} \Lambda^{k}\right) \mathbf{Q}^{H} \mathbf{d}
\end{array}
$$

where $\mathbf{Q}=\left[\mathbf{q}_{1}, \mathbf{q}_{2}, \ldots, \mathbf{q}_{K}\right]$ is a matrix containing orthonormal eigenvectors, with $\mathbf{q}_{1}=1 / \sqrt{K}[1 \cdots 1]^{T}$, and $\Lambda=\operatorname{diag}\left[\lambda_{1} \cdots \lambda_{K}\right]^{T}$ is the corresponding eigenvalue matrix

$$
\Lambda=\left[\begin{array}{cccc}
1 & 0 & \cdots & 0 \\
0 & 1-\frac{K c}{K-1} & 0 & 0 \\
0 & 0 & \ddots & \vdots \\
0 & 0 & \cdots & 1-\frac{K c}{K-1}
\end{array}\right] .
$$

To get the system stable all eigenvalues have to satisfy $\left|\lambda_{i}\right| \leq 1$, which means that the condition for stability becomes

$$
\lim _{n \rightarrow \infty}\left(1-\frac{K c}{K-1}\right)^{n}=0
$$

giving

$$
\begin{aligned}
\left|1-\frac{K c}{K-1}\right| & <1, \\
0 & <c<\frac{2(K-1)}{K} .
\end{aligned}
$$

When $c$ is chosen in the region of stability the mean value of the offsets becomes

$$
\begin{aligned}
E\{\boldsymbol{\theta}[n+1]\}= & \mathbf{Q} \Lambda^{n+1} \mathbf{Q}^{H} \boldsymbol{\theta}[0]+c \sum_{k=0}^{n} \mathbf{Q} \Lambda^{k} \mathbf{Q}^{H} E\{\mathbf{v}[n-k]\} \\
& +\mathbf{Q}\left(\sum_{k=0}^{n} \Lambda^{k}\right) \mathbf{Q}^{H} \mathbf{d} \\
= & \left(\sum_{i=1}^{K} \lambda_{i}^{n+1} \mathbf{q}_{i} \mathbf{q}_{i}^{H}\right) \boldsymbol{\theta}[0] \\
& +\left(\sum_{i=1}^{K}\left(\sum_{k=0}^{n} \lambda_{i}^{k}\right) \mathbf{q}_{i} \mathbf{q}_{i}^{H}\right)\left(c \mathbf{m}_{\mathbf{v}}+\mathbf{d}\right)
\end{aligned}
$$

where $\mathbf{m}_{\mathbf{v}}=E\{\mathbf{v}[n-k]\}$. The first term, which corresponds to the evolution of the initial offsets $\theta[0]$, becomes

$$
\begin{aligned}
& \lim _{n \rightarrow \infty}\left(\sum_{i=1}^{K} \lambda_{i}^{n} \mathbf{q}_{i} \mathbf{q}_{i}^{H}\right) \boldsymbol{\theta}[0] \\
& =\lambda_{1} \mathbf{q}_{1} \mathbf{q}_{1}^{H} \boldsymbol{\theta}[0] \\
& \quad=\left(\frac{1}{K} \sum_{i=1}^{K} \theta_{i}[0]\right) \times\left[\begin{array}{lll}
1 & \cdots & 1
\end{array}\right]^{T}
\end{aligned}
$$

which is the mean value of all modems start offset. The evolution of the second term in (26), corresponding to the effects of estimation errors of $\hat{\boldsymbol{\theta}}_{\mathrm{av}}[n]$ and the clock-drifts $\mathbf{d}$, depends on the powersum of the eigenvalues, $\sum_{k=0}^{n} \lambda_{i}^{k}$. For large $n$ they grow as

$$
\sum_{k=0}^{n} \lambda_{i}^{k} \approx \begin{cases}n, & i=1 \\ \frac{K-1}{K c}, & i \in\{2, \ldots, K\}, \quad n \gg 1 .\end{cases}
$$

Since the powersum of the first eigenvalue will be dominant when $n$ grows with time, the contribution from the first eigenvector, $\mathbf{q}_{1} \mathbf{q}_{1}^{H}=(1 / K) \mathbf{1}$, will dominate in (26). The clock-drift for each modem (relative to a perfect reference clock) will therefore converge to the average of all modems local clock-drift. However, all modems offsets can drift commonly relative to a perfect reference clock but this does not affect the orthogonality of the DMT symbols.

\section{REFERENCES}

[1] A. Ruiz, J. M. Cioffi, and S. Kasturia, "Discrete multiple tone modulation with coset coding for the spectrally shaped channel," IEEE Trans. Commun., vol. 40, pp. 1012-1029, June 1992.

[2] "Transmission and Multiplexing (TM); Access transmission systems on metallic cables; Very high speed Digital Subscriber Line(VDSL); Part 1: Functional requirements," ETSI, Tech. Spec. TS 101 270-1 V1.1.6 (1999-08), Aug. 1999.

[3] F. Sjöberg, M. Isaksson, R. Nilsson, P. Ödling, S. K. Wilson, and P. O. Börjesson, "Zipper: A duplex method for VDSL based on DMT," IEEE Trans. Commun., vol. 47, pp. 1245-1252, Aug. 1999.

[4] F. Sjöberg, R. Nilsson, M. Isaksson, P. Ödling, and P. O. Börjesson, "Asynchronous zipper," in Proc. Int. Conf. Communications, vol. 1, Vancouver, Canada, June 1999, pp. 231-235.

[5] P. Ödling, B. Mayr, and S. Palm, "The technical impact of the unbundling process and regulatory action," IEEE Commun. Mag., vol. 38, pp. 74-80, May 2000.

[6] P. J. Tourtier, R. Monnier, and P. Lopez, "Multicarrier modem for digital HDTV terrestrial broadcasting," Signal Processing: Image Commun., vol. 5, pp. 379-403, Dec. 1993.

[7] J.-J. van de Beek, M. Sandell, and P. O. Börjesson, "ML estimation of timing and frequency offset in OFDM systems," IEEE Trans. Signal Processing, vol. 45, pp. 1800-1805, July 1997.

[8] J.-J. van de Beek, M. Sandell, M. Isaksson, and P. O. Börjesson, "Low-complex frame synchronization in OFDM systems," in Proc. Int. Conf. Universal Personal Communications, Tokyo, Japan, Nov. 1995, pp. $982-986$.

[9] A. Peled and A. Ruiz, "Frequency domain data transmission using reduced computational complexity algorithms," in Proc. IEEE Int. Conf. Acoustics, Speech, Signal Processing, Denver, CO, 1980, pp. 964-967.

[10] "Transmission and Multiplexing (TM) access transmission systems on metallic access cables; Very High-Speed Digital Subscriber Line (VDSL) Part 2: Transceiver Specification," ETSI STC TM6, Sophia Antipolis, France, Tech. Spec. TS 101 270-2 V1.1.1 (2001-02), Feb. 2001.

[11] T. Pollet, P. Spruyt, and M. Moeneclaey, "The BER performance of OFDM systems using nonsynchronized sampling," in Proc. Globecom, vol. 1, San Francisco, CA, Nov. 1994, pp. 253-257. 
[12] M. Sandell, J.-J. van de Beek, and P. O. Börjesson, "Timing and frequency synchronization in OFDM systems using the cyclic prefix," in Int. Symp. Synch, Essen, Germany, June 1995, pp. 16-19.

[13] K. Jacobsen, "Methods of upstream power backoff on very high-speed digital subscriber lines," IEEE Commun. Mag., vol. 39, pp. 210-216, Mar. 2001.

[14] FSAN VDSL Working Group, "Power back-off methods for VDSL," ETSI TM6, Luleå, Sweden, Tech. Rep. 983t17, June 1998.

[15] T. Pollet and P. Timmermans, "Power back-off strategies for VDSL-TDD versus FDD, performance comparison," ETSI STC TM6, Sophia Antipolis, France, Tech. Rep. 985t24a0, Nov. 1998.

[16] F. Sjöberg, R. Nilsson, S. K. Wilson, D. Bengtsson, and M. Isaksson, "Power back-off for multiple target bit rates," ETSI STC TM6, Sophia Antipolis, France, Tech. Rep. 985t25a0, Nov. 1998.

[17] "Very-high-speed digital subscriber lines_-System requirements," ANSI, Plano, TX, Tech. Rep. T1E1.4/98-043R8, Nov. 1998.

[18] G. Forney and M. Eyuboglu, "Combined equalization and coding using precoding," IEEE Commun. Mag., vol. 29, pp. 25-34, Dec. 1991.

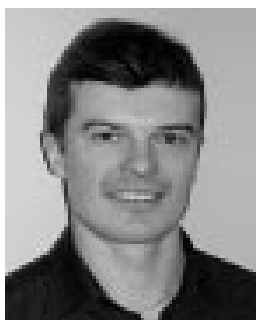

Rickard Nilsson (S'96) was born in Umeå, Sweden in 1971. He received the M.S. degree in computer science, the Lic.Eng. degree and the Ph.D. degree, both in signal processing, all from Luleå University of Technology in Luleå, Sweden, in 1996, 1999, and 2002 , respectively.

His research interests include multicarrier modulation, statistical signal processing and low-complexity algorithms for high-performance communication systems.

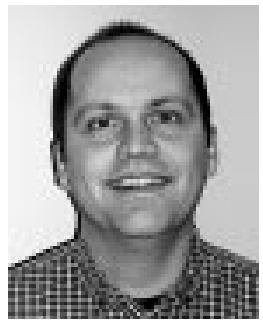

Frank Sjöberg (S'95-A'00-M'01) was born in Umeå, Sweden, in 1970. He received the M.S. degree in computer science and the Ph.D. degree in signal processing, both from Luleå University of Technology, Luleå, Sweden, in 1995 and 2000, respectively.

Since 2000, he has worked as a Research Engineer at Telia Research AB. He also holds a position as Assistant Professor at the Division of Signal Processing at Luleå University of Technology. His primary research interest is high-performance communication systems, with emphasis on wireline systems.

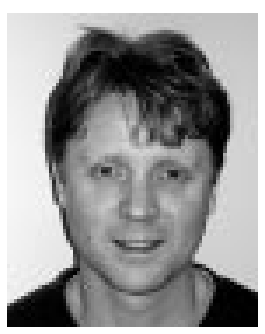

Mikael Isaksson received the M.S. degree in computer science from Luleå University of Technology, Sweden, in 1989.

Between 1989 and 2001, he worked at Telia Research $\mathrm{AB}$, the research branch of the Swedish incumbent telephone company, Telia AB. From 1989 to 1995 , he was active in research on radio communications. From 1995 to 2001, he was Project Manager at Telia Research for VDSL projects and active in ETSI, ANSI and ITU-T Committees for VDSL standardization. Since May 2001, he is the CEO and co-founder of UpZide Labs AB, a company active in the DSL area. He is author of over 15 patents in the fields of digital communication and signal processing, including the basic patents on the Zipper duplex method that is a part of the DMT-based VDSL standard in T1E1.4 and ETSI TM6.

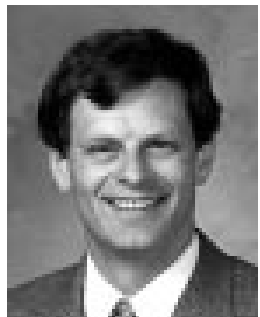

John M. Cioffi (S'77-M'78-SM'90-F'96) received the B.S.E.E. degree from the University of Illinois, Urbana-Champaign, in 1978, and the Ph.D.E.E. degree from Stanford University, Stanford, CA, in 1984

He was with Bell Laboratories, Holmdel, NJ, from 1978 to 1984 and IBM Research, San Jose, CA, from 1984 to 1986 . He has been with Stanford University as an Electrical Engineering Professor from 1986 to the present. He founded Amati Communications Corporation, Palto Alto, CA, in 1991 (it was purchased by Texas Instruments, Incorporated in 1997) and was Officer/Director from 1991 to 1997. He is currently on the boards or advisory boards of BigBand Networks, Coppercom, GoDigital, Ikanos, Ionospan, Ishoni, IteX, Marvell, Kestrel, Charter Ventures, and Portview Ventures. He is a Member of the U.S. National Research Council's CSTB. His specific interests are in the area of high-performance digital transmission.

Dr. Cioffi has received the following awards: the National Academy of Engineering in 2001, IEEE Kobayashi Medal in 2001, IEEE Millennium Medal in 2000, IEE JJ Tomson Medal in 2000, 1999 University of Illinois Outstanding Alumnus, 1991 IEEE COMMUNICATIONS MAGAZINE best paper; 1995 ANSI T1 Outstanding Achievement Award, and NSF Presidential Investigator from 1987 to 1992. He became a member of the National Academy of Engineering in 2001 He has published over 200 papers and holds over 40 patents, most of which are widely licensed, including basic patents on DMT, VDSL, and V-OFDM.

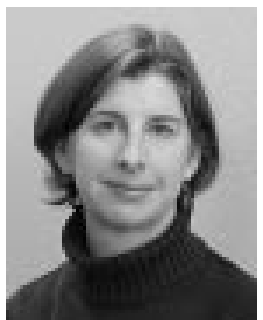

Sarah Kate Wilson (M'87-SM'99) received the A.B. degree in mathematics from Bryn Mawr College, Bryn Mawr, PA, in 1979 and the M.S. and Ph.D. degrees in electrical engineering from Stanford University, Stanford, CA, in 1987 and 1994, respectively.

She worked in industry from 1979 to 1989 , first as a Programmer/Analyst, then as a Research Engineer. From 1994 to 1999, she was an Assistant Professor of Electrical Engineering at Purdue University, West Lafayette, IN, and then at Luleå University of Technology, Luleå, Sweden. In August, 2000 she was awarded the title of Docent in Signal Processing (Associate Professor) from Luleå University of Technology. She is currently a Manager of Advanced WCDMA Research at ArrayComm, Inc. in San Jose, CA, and an Associate of Editor for IEEE TRANSACTIONS ON WIRELESS COMMUNICATIONS. 